teilweise Vernähung wenden wir grundsätzlich an. Es ist selbstverständlich, daß wir der Lage des Falles das Verfahren anzupassen haben. Je mehr der Erkrankungsherd lokalisiert und abgeschiossen war, je mehr wir also mit einer verhältnismäßig geringen Virulenz der Erreger und mit der Wahrscheinlichkeit rechnen können, alles Krankhafte entfernt zu haben, um so unbedenklicher und um so weiter dürfen wir schließen. Die genaue Berücksichtigung des einzelnen Falles wird uns aber auch manchmal abhalten, überhaupt $\mathrm{zu}$ nähen; wir lassen die ganze Wunde wenigstens eine Zeitlang offen. Nicht nur die Mitbeteiligung des Sinus oder des übrigen Schädelinhaltes werden uns dazu veranlassen. Nach diesen Grundsätzen haben wir seit Jahren gehandelt. Bis jetzt sehen wir auch keine Veranlassung davon akzugehen.

Nach teilweiser Naht und Offenhalten der unteren Wundwinkel heilen die Höhlen in der Regel ohne jegliche besondere Nachbehandlung. Wir können uns auf eine lockere Tamponade, die das Lumen der Höhle bis zum Versiegen der Absonderung offen hält, beschränken. Die Patienten können also bald aus der Klinik entlassen werden und bedürfen nicht unbedingt der Weiterbehandlung durch den Facharzt bis zur völligen Heilung. Als endgültigen Zustand, der für uns auch das beste Erreichbare darstellt, haben wir, wie ich durch eigene Untersuchung feststellen konnte, eine Ausfüllung möglichst großer Teile der Operationshöhle durch eine von den bedeckenden Weichteilen gelieferte Narbenbildung anzusehen. In der Tiefe, in der Antrumgegend, werden wir wohl immer mit erneuter Hohlraumbildung zu rechnen haben. Dort stellen sich unter günstigen Bedingungen, d. h. wenn die Verbindung mit der ausgeheilten Paukenhöhle erhalten bleibt, lufthaltige Räume wieder her. Pneumatische Räume in der Tiefe, feste Narbenbildung in den äußeren Partien der Operationshöhle, das stellt meines Erachtens das ideale Heilungsresultat dar.

Im allgemeinen bleibt das auch ein unveränderter Dauerzustand. Freilich ganz ausgeschlossen sind Rückfälle nicht. Es kommt dann bei erneuter Infektion der Paukenhöhle auch zu rasch auftretenden Entzündungen und Abscessen in der Operationshöhle und dem ausfüllenden Narbengewebe. In der Regel genügt dann ein Einschnitt in die vorgewölbte Narbe und das Offenhalten dieses Einschnittes, um die Entzündung wieder abklingen zu lassen. Neue große Operationen in Form von plastischem Ausfüllen der alten Höhle haben wir nicht mehr nötig gehabt, seitdem wir mindestens die obere Hälfte der Wunde durch Naht schließen, und seitdem wir durch Wegnahme des äußeren Teiles der hinteren knöchernen Gehörgangswand und möglicher Abflachung der Wundränder die Heilung im oben ausgeführten Sinne erstreben. Bei großen breiten Narben, womöglich noch mit Neigung zur Bildung von Dauerfisteln, ist aber ein plastisches Ausfüllen der Höhlen nicht zu umgehen.

\section{DIE UNTERSUCHUNG UND BEHANDLUNG VON STIMMSTÖRUNGEN DER REDNER UND SÄNGER.} Von

\section{Privatdozent Dr. M. NADOLECzNy, München.}

Obwohl die funktionelle Stimmschwäche schon seit I 862 (LewINs Arbeit in Virchows Archiv. Bd. 24) bekannt ist, und obwohl FIATAU und IMHOFER sie monographisch dargestellt haben, steht doch die Mehrzahl der Halsärzte dieser Krankheit - sagen wir höflich - ,etwas fremd" gegenüber. Dem sollte aber nicht so sein. Hat dieser unerfreuliche $\mathrm{Zu}$ stand doch zu einer stets wachsenden Antipathie der Stimmbildner gegen die Fachärzte geführt. Jene erlauben sich z. B. ganz unverfroren davon zu reden und zu schreiben, da $B$ die Halsärzte diesem Leiden ,kenntnis- und ratlos gegenüberstehen" und es den , unfruchtbaren, ja sogar oft stimmschädigenden Pinsel- und anderen Verfahren" unterwerfen. Leider sind solch übertriebene Vorwürfe wie dieser, dem Schriftchen eines Volksschullehrers entnommene, teilweise berechtigt. Aber es scheint auch erklärlich, daß ein großer Teil der
Laryngologen sich nicht recht in den Begriff der sogenannten Phonasthenie und in ihre Behandlung einzuleben vermag, - erklärlich durch die Art und Weise der ärztlichen Schulung in den zwei letzten Jahrzehnten des I9. Jahrhunderts, welche doch im wesentlichen von einer mechanistischen, also einer dogmatisch materialistischen Betrachtungsweise im Sinne der philosophischen Terminologie getragen war. Es würde freilich zu weit führen und aus dem Rahmen dieser $\mathrm{Ab}$ handlung fallen, wollten wir jenen'serkenntnis-theoretischen Zusammenhang weiter erörtern. Jedenfalls müssen die Halsärzte dem normalen und pathologischen Verhalten der Stimmfunktion, also der phonetischen Seite der Laryngologie, im eigenen Interesse und in dem ihrer Kranken mehr Beachtung schenken, als das bisher wenigstens in der Praxis geschah.

Bevor wir das Thema der Aufschrift im einzelnen behandeln, soll der Begriff der funktionellen Stimmschwäche begrenzt werden, als ,Störung, deren Wesen darin besteht, daß die Lautgebung beim berufsmäßigen Sprechen und Singen nicht mit einem gewohnheitsgemäßen und daher unbemerkten Aufwand an Kraft, nicht mit gewohnter Klangreinheit und nicht in gewahnter Dauer hervorgebracht werden kann".

Der Vorgeschichte unserer Kranken (Prediger, Berufsredner, Lehrer an Volks- und Mittel-, selten an Hochschulen, Schauspieler, Sänger) soll hier nur weniges Bedeutsame entnommen werden. Die meisten kommen mit einer fertigen Diagnose zum Arzt: Rachen- Kehlkopf-, Luftröhrenkatarrh oder alle drei , Katarrhe" zusammen. Auf die Frage nach dem Auswurf hört man mit Erstaunen, daB trotz jener Katarrhe kein oder fast kein Schleim ausgespuckt werden könne, obwohl er da sei. Die Katarrhhypothese fuBt, soweit sie nicht ärztlicherseits ausgesprochen wurde, auf subjektiven Empfindungen aller Art vom einfachen Druck, von der Trockenheit bis zum brennenden Schmerz. Die Stimme, welche früher mühelos ansprach, wird jetzt rasch belegt, verschleiert, überhaucht, heiser. Singen und Sprechen strengt an und ermüdet schon nach $1 / 4$ oder $1 / 2$ Stunde. Die Singstimme hat anfangs namentlich in der Mittellage gelitten, in der Höhe erst später, aber auch hier brechen die Töne ab bzw. sind zum Teil verloren gegangen. Beim längeren Sprechen und Singen verschlechtert sich der Zustand, der gewöhnlich sehr allmählich, selten plötzlich im Anschlu $\beta$ an eine besondere Leistung oder an eine akute Erkrankung sich entwickelt hat. Das plötzliche Auftreten findet man häufiger bei zu kurz und ungenügend geschulten Sängern. Doch lehrt uns genaueres Nachfragen, daß auch hier schon vorher Anzeichen der beginnenden Störung da waren, die nicht genügend gewürdigt wurden. Deshalb hat sich die Aufnahme der Vorgeschichte, abgesehen von den erwähnten Beschwerden, noch zu erstrecken auf den Gesang in der Schule, während der Pubertät, auf den Eintritt der letzteren (erste Menstruation), auf Mutationserscheinungen und Mutationsdauer, ferner auf die gesangliche Ausbildung, deren Dauer und Art (wie lange täglich; wie viel Jahre, was für Utbungen, wann zuerst Iieder, Partien gesungen wurden [zu früh ?] und welche). Von besonderer Wichtigkeit ist auch die sogenannte Gesangsmethode. Man muß also wissen ob mehr forte (falsch) oder mehr piano, auf welche Vokale hauptsächlich geübt wurde. ferner was von Stimmeinsatz, Stimmansatz und Atmen gelehrt wurde, ob offenes Singen besonders (zu viel) gepflegt und ob die Stimme hinaufgeschraubt wurde. Nach diesen Vorfragen beginnt erst die eigentliche Vorgeschichte mit Fragen nach der ersten, etwaigen späteren und schließlich der gegenwärtigen Stimmstörung, deren Art (Tongebiet) und angebliche Ursachen (Krankheit, Überanstrengung, seelischer Schock) und schließlich deren Behandlung bzw. die therapeutische Mißhandlung (örtliche Eingriffe mit Ätzmitteln, Kaustik oder Operationen, deren Zweckmäßigkeit oder Schädlichkeit hinterher oft schwer zu beurteilen ist). Die subjektiven Angaben des Kranken sind hier wenig maßgebend; objektive Befunde wie $z$. B. vollkommene Entfernung der unteren Muscheln, oder Synechien nach Kanstik in der Nase sagen uns mehr.

Die örtliche Untersuchung und ihr Ergebnis, der Befund, ist bei Phonasthenie recht häufig fast belanglos: man findet nichts besonderes und was man findet, $z$, B. eine Septum- 
verbiegung oder eine Spina, darf nicht allzu hoch im Sinne einer Ursache bewertet werden. Bei ganz akuten frischen Fällen läßt sich eine stärkere Rötung der Stimmlippenränder oder auch der ganzen Stimmlippen feststellen. Dies oder gar eine frische Blutung gehört aber schon nicht mehr hier ins Gebiet der Phonasthenie. Interarytänoidale Rötungen, sowie leichte Randrötung der Stimmlippen sind dagegen häufige Folgen der Erkrankung namentlich des allzu vielen Räusperns und Hüstelns, dazu gehören auch Rötungen der Rachenhinterwand und der Seitenstränge, die mehr oder minder belanglos sind. Wenn irgendwo, so müssen wir hier im Sinne von J. FEIN den wahren und den falschen Rachenkatarrh unterscheiden. Zu ersterem gehört der Nachweis von Sekret, das besonders aus den mittleren Spalten des Rachendaches kommt, hinten herunter fließt, ausgespuckt wird, und am ehesten in Morgenstunden gesehen werden kant.

Wichtiger ist es, die örtlichen Empfindungen der Kranken, die Parästhesien (Trockenheit-, Sand-, Kratz-, Brenn-, Schleim-, Druckempfindungen), zu berücksichtigen und nachzusehen, von wo sie ausgelöst werden. Daher ist es zweckmäßig, sich vom Kranken am Hals außen die Stelle zeigen zu lassen, wo er die unangenehmen Empfindungen lokalisiert. Man kann dann mit Sicherheit annehmen, daß sie innen im Hals ungefähr um eine Handbreite höher wahrgenommen werden, eine Erscheinung, auf die Herrmann KRAust hingewiesen hat. Wenn man nun mit einer Sonde die entsprechenden Stellen der Rachenhinterwand leise berührt, womöglich ohne den Rachenreflex auszulösen, so gibt der Untersuchte meist mit dem Ausdruck der Befriedigung an, das sei genau die Stelle, wo es ihn kratze oder drücke. So lrommt man fast immer bei solchen Empfindungen auf die Mitte der Rachenhinterwand, bei einseitigen Schmerzen auf die Seitenstränge. Findet der Kranke, man berühre zu tief, so liegt der Ort der Parästhesie am Rachendach, meint er, man berühre zu hoch, so trifft man das richtige, wenn man mit der Sonde gegen den Kehlkopfeingang sich bewegt. In dieser Gegend werden die allermeisten Parästhesien lokalisiert. Das ist nun eine bekannte Tatsache, aber man sollte sich nicht mit dem nichtssagenden Wort Globusgefühl oder gar Globus hystericus zufrieden geben. Die Gegend des Plexus pharyngeus ist ausgezeichnet durch einen besonderen Empfindungsreichtum, und mancher seelische Schmerz wird dort örtlich projiziert, nicht nur der Schmerz beim unterdrückten Weinen, die zusammenschnürende Empfindung der Angst. Ich erinnere mich eines jungen schneidigen Offiziers, der im Anschluß an den plötzlichen Tod seines besten Freundes (Absturz in den Alpen, vor dem Krieg!), den er nicht verhindern konnte, mehrere Tage lang heftige schneidende Halsschmerzen hatte, die genau an der Rachenhinterwand lokalisiert waren und ohne örtliche Behandlung verschwanden, nachdem der Leidende Aufklärung über das Wesen des Schmerzes erhalten hatte. Dieser AufschluB kam ihm um. so überraschender, da er mir den Unglücksfall verschwiegen hatte. Eine recht häufige Ursache für Parästhesien der Rachens, besonders für Druckempfindungen, ist ferner die Obstipation, und zwar die spastische. Andere Empfindungen, die tiefer wahrgenommen werden, sind meist an örtliche Erkrankungen des Kehlkopfes (lokalisiert in der Gegend unter dem Schildknorpel) oder der Luftröhre (lokalisiert am Brustbein) geknüpft. Doch kommen bei Sängern die letzteren auch ohne örtlich nachweisbare Krankheiten vor als Begleiterscheinung von Ermüdungszuständen. Echte Schmerzen in der Kehlkopfgegend bei Neuralgikern sind kenntlich am Druckpunkt des Laryngeus superior, werden aber doch oft falsch gedeutet. Sie sind gar nicht selten mit Neuralgien im Trigeminus- und Occipitalisgebiet vergesellschaftet und können auch akute infektiöse Erkrankungen des Rachens begleiten. Der phonasthenische Schmerz ist also durchaus nichts Eindeutiges; bevor man ihn annimmt, muß man andere Formen ausschließen. Daß auch das Allgemeinbefinden nicht unberücksichtigt bleiben darf, daB auf Herz- und Lungenkrankheiten, allgemeine Nervosität, etwaige Anämie oder Chlorose geachtet werden $\mathrm{mu} B$, ist selbstverständlich. Eine besondere Rolle spielt in diesem Zusammenhang die spastische Obstipation nicht nur wegen der Parästhesien in den oberen
Luftwegen (Rachen und Nase), sondern auch wegen der von der Darmstörung abhängigen Schwellungszustände der Nasenschleimhaut, die kausal; nicht örtlich behandelt werden sollte.

Die funktionelle Untersuchung ist der wichtigste Teil unserer Tätigkeit beim Aufbau der Diagnose. Sie erfordert vom Arzt ein wenigstens mittelmäßiges musikalisches Gehör und ein auf Klangeigenschaften eingeübtes $\mathrm{Ohr}$. Wer sich nicht einigermaßen auf diese Dinge einschulen kann, derlasse die Hände von der Beurteilung und der Behandlung der Stimmstörungen. Je mehr musikalische Veranlagung und Kenntnisse der Arzt hier mitbringt, desto leichter wird er sich einarbeiten. Außer in ganz akuten oder sehr schweren Fällen ist die Sprechstimme der Umgangssprache nicht eigentlich heiser, wohl aber oft leicht belegt, und meistens fallen schon beim gewöhnlichen Reden harte Stimmeinsätze auf. Ihre ungefähre Tonlage muß bestimmt werden. Das Bild ändert sich sofort, wenn man im Vortragston vorlesen oder vortragen läßt, wie es z. B. der Lehrer in der Schule tut. Die Anstrengung dabei wird augenfällig, die Tonlage erhebt sich wesentlich (um eine Terz oder Quart) über die ruhige Tongebung der Umgangssprache. Die Aussprache erscheint verändert durch die häufige Übertreibung des Stärkeakzents, die harten Stimmeinsätze werden für den Zuhörer fast unerträglich. Die Singstimme muB man auch beim Redner prüfen. Da entdeckt man dann bisweilen $z$. B., daß ein tiefer $\mathrm{Ba} B$ beim Unterricht in Tenorlage kräht. Den Umfang der Singstimme sollte man stets bei ganz leiser Tongebung bestimmen, nicht ohne sich nach dem früheren Stimmumfang erkundigt zu haben, um so zunächst die Zahl der hohen Töne festzustellen, die verloren gingen. Alle anderen Fehler der Tongebung lassen sich beim Pianound Pianissimosingen ebenfalls leichter beobachten, während beim Forte der Sänger uns über viele Mängel hinwegtäusçhen kann. Man muß besonders auf folgende Fehler achten und notieren, bei welchen Tönen bzw. in welchen Tonbereichen sie vorkommen: Detonieren (nach unten) oder Distonieren (nach oben) beim Toneinsatz, beim Tonhalten, beim Ausklingen eines Schwelltons, Reibegeräusche beim Stimmeinsatz (Knacken), überhauchte Tongebung (sogenannte ,, wilde Luft" oder ,Faden in der Stimme") Flattern länger gehaltener Töne, Tremolieren, Abbrechen der Töne (sowohl beim Einsetzen wie auch besonders beim Abschwellen, namentlich der sogenannten Ubergangstöne). Stets sollte zunächst der ganze Tonbereich geprüft werden, d. $h$. beim Sopran die Töne a $h$ bis $a^{2} c^{3}$, beim Mezzosopran a bis $a^{2}$, beim Alt $f$ bis $f^{2}$, beim Tenor $A H$ bis $a^{1} c^{2}$, beim Bariton $F$ bis $f^{1}$, beim $B a B E$ bis $e^{1}$. Die Registergrenzen lassen sich beim Aufwärtssingen im Piano durch den ganzen Stimmumfang gewöhnlich heraus hören. Eingehender sind die sogenannten Übergangstöne (an den Registergrenzen) zu prüfen, nämlich meistens beim $B a \beta c$ d, $\mathrm{c}^{\mathbf{1}} \mathrm{d}^{\mathbf{1}}$, beim Bariton $\mathrm{d} e, \mathrm{~d}^{\mathbf{1}} \mathrm{e}^{\mathbf{1}}$, beim Tenor e $\mathrm{f}, \mathrm{e}^{\mathbf{1}} \mathrm{f}^{\mathbf{1}}$, beim Alt und Mezzosopran $\mathrm{e}^{\mathbf{1}} \mathrm{e}^{\mathbf{2}}$, beim Sopran $\mathrm{e}^{\mathbf{1}} \mathrm{f}^{1}, \mathrm{e}^{\mathbf{2}} \mathrm{f}^{\mathbf{2}}$ und zwar im piano, im forte und bei Schwelltönen. Auf denselben schwierigen Tönen sind auch alle Vokale zu prüfen, ferner der Übergang von tönenden Konsonanten zum Vokal z. B. w-a, w-o usw., schließlich die GutzMANNsche Druckprobe: ein Sington, der, während man einen leichten Druck auf den Schildknorpel ausübt, längere Zeit gehalten wird, weicht für Bruchteile von Sekunden nach oben ab, wenn der Druck auf den Schildknorpel plötzlich aufgehoben wird, worauf der Singende sofort wieder zur ursprünglichen Tonlage im normalen Falle zurückkehrt, während der Stimmkranke jene ursprüngliche Tonlage nicht gleich oder gar nicht wiederfindet. Auffäliigere Abweichungen von der Norm bei dieser Probe kommen indes nur bei schwereren Stimmstörungen vor. Die funktionelle Prüfung ist erst beendet, wenn man sich auch über die Klangfarbe in den verschiedenen Tonlagen klar geworden ist, obwohl hierbei ästhetische Werturteile nicht $\mathrm{zu}$ vermeiden sind, über die man bekanntlich verschiedener Meinung sein kann. Immerhin ist es zweckmäBig zu beachten ob die Töne voll, hart, offen, belegt, heiser, gaumig, kloßig, gepreßt oder hohl, ob sie offen oder geschlossen nasal klingen (Probe auf die Vokale A und I mit offener und zugehaltener Nase). Die Feststellung akustischer Erscheinungen geschieht am Klavier oder noch besser durch Vergleich 
mit Stimmgabeltönen z. B. der BEzoLD-EDELMANN schen Tonreihe. Letzteres hat den Vorteil, da $B$ der Singende den Ton nur im Anfang hört, wenn ihm die leise klingende Gabel ans Ohr gehalten wird. Dann wird er sich selbst überlassen. Die Klaviertöne erlauben ihm dagegen während des Singens seine Leistung durch Vergleichen unter Kontrolle $\mathrm{zu}$ balten, wodurch ihm die Möglichkeit bleibt, den Untersucher zu täuschen.

Die Untersuchung wäre nicht vollständig, würde man die sichtbaren und meßbaren Bewegungen des Phonationsapparates außer acht lassen. Die Atembewegungen sind zwar der einfachen Betrachtung zugänglich, genaueren AufschluB über ihren Ablauf im einzelnen gibt uns erst die pneumographische Aufnahme von Brust- und Bauchatmung. Solche Kurven, auf denen die Ruheatmungskurve nicht fehlen darf (zum Vergleich), sollten immer vom gleichen Sprechbeispiel, von denselben Tonfolgen aufgenommen werden. Mit dem Kehlkopfspiegel können wir die Einsatzbewegung der Stimmlippen beobachten. Äußere Kehlkopfbewegungen verfolgen wir mit dem Auge und dem Getast. Bewegungen des Gaumensegels und der Zunge (immer bei verschiedenen Tönen) sehen wir im Lichte des Reflektors ohne instrumentelle Hilfe, die Kieferöffnung und die Lippenbewegungen (Vokalstellung, Lippenzittern beim Singen), sowie Mitbewegungen des Gesichts und des ganzen Körpers dürften der einfachen Betrachtung nicht entgehen.

Endlich muß man sich einigermaßen über das musikalische Gehör der Kranken unterrichten, auch beim Redner, nicht nur beim Sänger. Je besser es beim Redner entwickelt ist, desto leichter wird er beim Úben die richtige Tonlage finden und festhalten können. Für den Sänger ist das musikalische Gehör natürlich ausschlaggebend. Wer bei einfachen Tonleitern, bei auf- und absteigenden Terzenfolgen, bei gebrochenen Akkorden, bei Tonleitern in Oktavsprüngen regelmäßig aus der Tonart fällt ohne es zu merken, der sollte den Beruf des Sängers aufgeben. Dasselbe gilt übrigens von den zahlreichen körperlich schwächlichen Gesangsschülerinnen mit typisch asthenischem Habitus und meist mäßigen Stimmmitteln, die ewig studieren und es nie zu etwas bringen. Ihnen abzuraten ist eine unserer unangenehmsten, aber ernstesten Pflichten. Dieser gutgemeinte Rat wird von unseren Patienten indessen gewöhnlich zu spät befolgt, naclidem sie schon große Summen für Ausbildungszwecke verloren haben. Dagegen ist das Detonieren und dann in der anderen Tonart weitersingen noch als phonasthenisches Zeichen zu werten.

Die Untersuchung von stimmgestörten Rednern und Sängern ist also wirklich keine einfache Sache, die man im Handumdrehen erledigen kann. Will man seine Arbeit ordentlich machen, so ist es zweckmäßig sich an ein Untersuchungsschema zu halten, das zwischen den Zeilen der obigen Ausführungen steht. Ferner gehört neben psychologischem Verständnis und phonetischen und musikalischen Kenntnissen doch auch eine gewisse Erfahrung dazu, die sich jeder erarbeiten muß, und schließlich recht viel Geduld.

Geduld erfordert dann erst recht die Behandlung. Es ist nicht unberechtigt die Frage zu stellen, ob man überhaupt örtlich behandeln dürfe. Jedenfalls sollte man es nicht in der Art tun, wie es bisher vielfach geschah. Die Nasen der Phonastheniker sind nicht der Ort, um sich rhinochirurgisch auszutoben, und ihre Mandeln sind kein geeignetes Objekt zu Ausschälübungen. Auch Jod-, Höllenstein-, Protargol- und Turiopinlösungen tun ihnen nicht gut und selbst Menthol in stärkerer Konzentration wird nicht immer vertragen. Die Anwendung dieser reizenden Mittel bei akuten Entzündungen ist ja schon strittig. Vielleicht wird eine Zeit kommen, in der wir akute Schleimhautentzündungen nach BLEULER mit Udenotherapie oder wenigstens mit reizmildernden Mitteln behandeln. SPIEss hat schon vor i6 Jahren (I906) auf die Bedeutung der Anästhetica (Anästhesin und Orthoform) hingewiesen. Sine Bihandlungsart scheint mir bei den Reizzuständen der Phonastheniker, bei der geringen Hypersekre- tion, dem sogenannten Ermüdungskatarrh bis heute nicht überholt. Gegen Schmerzen kommt noch trockene Wärme in Betracht. Ortliche Reize: zu viel Gurgeln, Räuspern, häufiges Leerschlucken sind zu untersagen. Menthol ist sehr verdünnt in Salbenform wohl meist unschädlich für Nase und Nasenrachen. Als Inhalationsmittel eignet sich noch am ehesten Aqua Laurocerasi (verdünnt). Die Allgemeinbehandlung ist nicht weniger wichtig als die örtliche. Namentlich ber ücksichtige man die spastische Obstipation und verbiete in solchen Fällen Nicotin und Tee strengstens. Alkohol ist bekanntlich schädlich. Wenn es sich aber um Phonastheniker ohne Verdauungsstörungen handelt, so kann man unter Umständen diese Gifte in sehr mäBigen Dosen erlauben, wenn allzuviel Verbote die Kranken zu ängstlich machen würden. Die seelische Beeinflussung derselben ist Sache der Erfahrung. Man muB sie aufrichten und das geht Hand in Hand mit der Ubungsbehandlung. Wichtig ist ferner daß man ihnen abgewöhnt sich darum zu kümmern, ob ihre Stimmlippen "schließen" und ob sie ,woiß" sind. Berufsredner und Sänger haben öfter stärker durchblutete Stimmlippen mit erweiterten Venen, das ist kein besonderer Befund. Bei Sängern, namentlich aber auch bei Lehrern handelt es sich nun meistens um die Existenz. Die schlimmen Erfahrungen mit örtlichen Behandlungen oder mit neuen Singmethoden, die ihnen nichts nützten, haben sie miBtrauisch gemacht. Selbst wenn sie sich schonen und nur bei besonderen Arlässen (z. B. Glanzstellen von Rollen) mit der Stimme herausgehen und damit die Zuhörer und sich selbst zu täuschen versuchen, so nagt doch die Sorge an ihrem Gemüt und läßt allmählich eine Erwartungsneurose anwachsen, die aus den zahlreichen Mißerfolgen gut erklärbar ist. Der Gesangsschüler wechselt den Lehrer, der Sänger den Arzt, der Lehrer behandelt sich oft selbst mit allerlei Kuren; Angst vor "Kehlkopfschwindsucht" und ,Krebs" sind nicht selten bei ihnen. Je genauer man sie untersucht, desto eher erwirbt man ihr Vertrauen, desto leichter kann man sie beruhigen. Statt also von nicht schließenden Stimmlippen zu reden, kläre man sie über die Ursachen ihrer Leiden auf. Einige Anästhesineinblasungen in den Nasenrachenraum, helfen zunächst über unangenehme Empfindungsstörungen namentlich der Gewohnheitsräusperer hinweg und erleichtern einem dabei im Anfang die funktionelle Behandlung.

Mit der Übungsbehandlung also mit der kausalen Therapie, soll man nämlich gleich anfangen, Schweigekuren wie bei akuter Laryngitis sind hicr VogelstrauBpolitik. Der Erfolg ist im besten Fall vorübergehend. Die einzelnen Teilvorgänge der Stimmgebung können getrennt oder gleich zusammen geübt werden je nach der Eigenart und Schwere des Falles. Mit Atemübungen mu $B$ man eine rasche geräuschlose tiefe Einatmung (Bauch- und Brustatmung), ohne Mitbewegungen (des Kopfes oder der Schultern), eine langsame gleichmäßige auf $\mathbf{I}_{5}$ bis $20 \mathrm{Sec}$. mindestens ausgedehnte Ausatmung erreichen. Stimmübungen müssen mit weichem Stimmeinsatz gelernt werden. Macht das Schwierigkeiten, so gehe man vom gehauchten zum geflüsterten Stimmeinsatz über oder lasse leise auf einen tönenden Konsonanten , $m$ " oder , $\mathrm{n}$ " summen. Man geht dabei von Tönen der Brust:timme oder der unteren Mittellage aus nach unten und oben und schreitet zweckmäßig nach der Höhe nicht $\mathrm{zu}$ schnell fort. Ist der Tonumfang in einiger Ausdehnung und leiser Stimmgebung. geübt, so darf man allmählich stärkere Tongebung erlauben. Fast immer gelingen die Utbungen anfangs am besten auf den Vokalo. Aber auch die übrigen Vokale müssen mit deutlichen Mundstellungen geübt werden und schließlich rein klingen. Hand in Hand mit der guten Aussprache der Vokale geht jene der Konsonanten. Beim Redner ist es auch nötig, die Stimmübungen nach oben auszudehnen, aber er muß vor allem lernen, mit guten Brusttönen zu sprechen. Sein Beruf verlangt neben deutlicher Aussprache eine sonore Stimmeam unteren Endedes Stimmumfangs (zwischen A und $\epsilon$ ). De; Sängers Stimme soll durch den ganzen Stimmumfang ausgeglichen klingen. Um das zu erzielen muB die Ubungsbehandlung schließlich zur künstlerischen Ausbildung hin- 
überleiten, die nicht mehr Sache des Arztes ist. Die ärztliche phonetische Therapie kann unterstützt werden durch (womöglich in ihrer Frequenz der Stimmhöhe angepaßte) elektrischeVibrationen und Ströme (vorzuziehen ist der pulsieren Gleichstrom). Beide Hilfsmittel sollen die Ubungsbehandlung begleiten als passive neben der aktiven Gymnastik. Ohne Stimmübungen scheinen sie wenig nutzbringend und können allenfalls nur als Suggestivmittel in Betracht kommen. Das Mucksche Kugelverfahren mag wohl eine psychogene Heiserkeit beseitigen, eine falsch eingeübte Funktion dürfte aber durch diesen Eingriff nicht in eine richtige umzustellen sein, eine Technik muB eben gelernt werden. Die Besserungen der Stimmfunktion während der Anwendung von schwachen elektrischen Strömen, Vibrationsmassage und Druck, also der sogenannten Ausgleichsverfahren sind prognostisch wertvoll. Die Methodik des Verfahrens ist bei FlataU oder ImHOFER nachzulesen und auf Grund der dortigen Angaben auszubauen bzw. dem einzelnen Fall anzupassen. Allerdings darf man die Vorhersage nicht nur auf den Ausfall solcher Ausgleichsversuche stellen. Die Dauer der Störung, das körperliche Befinden und das Alter der Kranken, ihre nervöse und seelische Verfassung, neurotische Zeichen und ihre Eignung zur Utbungsbehandlung (Verständnis, Geduld, musika- lisches Gehör) und nicht zuletzt der Aufwand an Zeit, den sie dafür machen können, sind dabei sehr maßgebend.

Die Beurteilung und Bshandlung der Phonasthenie ist, wie aus all dem hervorgeht, umständlich. Die Zustände, welche wir sehen sind außerordentlich mannigfaltig und verschieden nach Ursache und Auswirkung. Wenn wir trotzdem einen Durchschnittstyp des Phonasthenikers aufstellen sollen, so wäre es für den Redner jener, der zu hoch, mit harten Stimmeinsätzen und unsorgfältiger Artikulation spricht, und meist ein Gewohnheitsräusperer ist; für den Sänger, der ungenügend ausgebildete, mit mangelhafter Atemtechnik, der zu früh schon an schwierige Leistungen heranging, dessen Stimme nicht ausgeglichen (Registerausgleich), dessen Mittellage flach und überhaucht klingt und dessen Höhe in pianissimo versagt. Diesen Kranken können wir durch Maßnahmen helfen, welche sich unter der Bezeichnung phonetische Therapie zusammenfassen lassen. Die sachgemäße Anwendung solcher Verfahren ist aber auch das beste Mittel zur Bekämpfung der neuerdings auf diesem Gebiet sich immer breitermachenden Kurptuscherei.

Aus diesen Gründen ist es nötig, daß an den Universitätskliniken die Pathologie und Therapie der Stimmstörungen ebenso wie die der Sprachstörungen gelehrt wird.

\section{ÖFFENTLICHES GESUNDHEITSWESEN.}

\section{SOZIALHYGIENISCHES AUS DER STUDENTENSCHAFT.}

\author{
Von
}

Dr. med. F. Golddmann-Betlin.

Man hat in letzter Zeit befürchten müssen, die Eignung zum Besuch einer Hochschule würde bald nur noch durch die Größe des väterlichen Auslandsguthabens erwiesen werden. Wie weit das berechtigt ist, ergibt sich aus einem Blick auf die nachstehende Tabelle über die soziale Gliederung der Studentenschaft nach Berufsstand des Vaters, die bei der letzten bekannt gewordenen wissenschaftlich einwandfreien Erhebung, der an der technischen Hochschule Dresden vom Mai 1920, gewonnen wurde. Unter 1667 $=92 \%$ sämtlicher Studierender, waren die Väter:

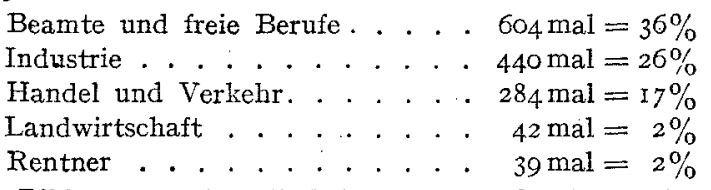
Diesem Bilde entsprechen die beiden anderen Umfragen der Nachkriegszeit, die Leipziger und Münchener aus dem Jahre 1920, nur sind sie infolge geringer Beteiligung bei der Beantwortung für sich allein nicht beweiskräftig. Also das, was aus Vorkriegserhebungen bereits bekannt war. Mehr als ein Drittel aller Studenten stammen aus dem Beamtentum (überwiegend aus dem niederen) und aus sozial ihnen gleichstehenden Schichten der Bevölkerung. Da die Beamten ein hygienisch recht gutes, ja zum Teil, ,erlesenes" Material darstellten, ist ihr wirtschaftliches Versinken auch vom gesundheitlichen Standpunkte nicht angenehm, zumal im Kriege zweifellos eine große Zahl körperlich oder geistig Minderwertiger freie Bahn zum sozialen Aufstieg hatten. Die besonderen wirtschaftlichen Schwierigkeiten dieser Bevölkerungsklassen spiegeln sich mit grausamer Deutlichkeit in den Einkommensverhältnissen der Studenten wieder.

Von den 865 männlichen Vollstudierenden deutscher Staatsangehörigkeit, die im Mai 1920 auf die Umfrage der Dresdner technischen Hochschule an tworteten, hatten ohne Nebeneinnahmen einen Monatswechsel von

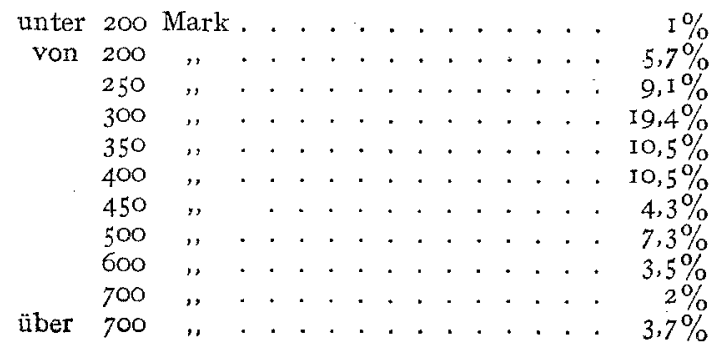

$\mathrm{Zu}$ gleicher Zeit wurde das monatliche Existenzminimum in Dresden auf $683 \mathrm{Mk}$. beziffert. In Leipzig stellte sich das Durchschnittseinkommen mehr als eines Drittels sämtlicher Teilnehmer am Zwischensemester 1920 auf monatlich $200-300 \mathrm{Mk}$., blieb also hinter dem zu damaliger Zeit von der Stadt Leipzig an einen ungelernten I 9 jährigen männlichen Arbeiter laut Tarif zu zahlenden Mindestlohn von $800 \mathrm{Mk}$, dem Existenzminimum, um $75-62 \%$ zurück. Im Herbst 1920 betrug der errechnete Durchschnittswechsel der Münchener Studenten $600 \mathrm{Mk}$. monatlich, eine Summe, die bereits ein Jahr vorher für München das Existenzminimum dargestellt hatte. Im Sommersemester 192 I mußte auf Grund von Auskïnften der einzelnen Universitäten bei einer Gresamtzahl von etwa I Io ooo Immatrikulierten die Zahl derer, die das ortsnotwendige Existenzminimum ohne Nebenerwerb nicht erreichten, auf etwa 80 ooo geschätzt werden, und unter ihnen hatten etwa 30 ooo ein Einkommen von weniger als $350 \mathrm{Mk}$. monatlich.

Diese Verhältnisse muBten die Lebenshaltung der Studentenschaft zunächst so gestalten, daß der Student seine bewährte Kunst, die Ausgaben auf ein Mindestmaß herabzudrücken, bis zur Virtuosität trieb, und zwar in erster Linie bei den ihn besonders belastenden Posten: Wohnung and Nahrung.

Leider sind brauchbare Unterlagen über die studentische Wohnungs- und Frnährungsfrage kaum vorhanden. Gerade auf dem studentischen Wohnungsmarkt mußte sich Fehlen von Räumen und mangelhafte Vermittlung nach Kriegsende stark fühlbar machen. In der Tat diente manchem in Berlin das Asyl für Obdachlose, ein Bahnhofswartesaal, eine Denkmalstufe. Vielen ein völlig unzureichendes Gelaß vorübergehend als Quartier. Bis sich überall durch Einrichtung studentischer Wohnungsämter und die bekannte Reklame in der Offentlichkeit Hilfe schaffen ließ. Preissenkung bei gewerbsinäßigen Vermittlern — Berlin lehnt die Vermittlungen von Zimmern, die mehr als $350 \mathrm{Mk}$. kosten ab - Ablösung von Zwangseinquartierung durch Aufnahme von Studenten, Errichtung von Wohnbaracken, Gründung von Siudentenheimen, vor allem aber Beschaffung von zahlreichen Unterkünften bei Privaten gegen geringe Bezahlung, gegen Arbeitsleistung oder unentgeltlich, ermöglichen jetzt wohl in den meisten Fällen Unterbringung in einer Umgebung, die geistiger Arbeit nicht ganz ungünstig ist. Vielleicht ist gerade durch die nicht geringe Anzahl von durch Private angebotenen Zimmern das hygienische Niveau der Studentenwohnung gehoben worden, und möglicherweise erklärt sich so auch das auffallend günstige Resultat der von der Universität Kiel im Sommersemester 1920 veranstalteten Umfrage. Sie ist die einzige Arbeit aus der Nachkriegszeit, die sich eingehender mit der Studentenwohnung beschäftigt. Die 1180 Angaben, die einer Beteiligung von $86 \%$ der Auswärtigen entsprechen, äußern sich über folgende Punkte: Ein Luftraum von weniger als $20 \mathrm{cbm}$ pro Person war nur in $1,1 \%$, Luftraum von mehr als $60 \mathrm{cbm}$ in $32,4 \%$, zwischen 20 und $60 \mathrm{cbm}$ in $46,3 \%$ vorhanden. Uber $2 / 3$ aller Zimmer hatten zwei und mehr Fenster. Die Tagesbeleuchtung, gemessen nach der Entfernung vom Fenster, in der in Tischhöhe noch Himmel zu 